\title{
Similarity Measure Based on Distance of Dual Hesitant Fuzzy Sets and Its Application in Image Feature Comparison and Recognition
}

\author{
Shihong Chen ${ }^{1, *}$ and Zhi-yong Bai ${ }^{2}$ \\ ${ }^{1}$ College of Applied Arts and Science, Beijing Union University, 197 Beitucheng West Rd., Beijing, 100191, China \\ ${ }^{2}$ College of Arts and Design, Beijing Forestry University, 35 Tsinghua East Road, Beijing, 100083, China
}

\begin{abstract}
In this paper, we propose the distance-based similarity measure of dual hesitant fuzzy sets (DHFSs) and apply it to the image feature comparison and recognition. Based on distance-based similarity measure of DHFS, we establish a comprehensive assessment method of image features to compare the images, where five criteria are represented by DHFSs to assess the image features. By the proposed method, we can determine ranking order of the six alternative images. The assessment results show that the proposed method is simple and effective to solve the problem of image feature comparison and image recognition, and provides a new assessment method for computer image processing experts.
\end{abstract}

Keywords: Image feature comparison, Image recognition, Image processing, Dual hesitant fuzzy set, Distance-based similarity measure.

\section{INTRODUCTION}

Fuzzy sets (FSs) provide human-like making system in an intuitive way and convert system specifications into effective models. Zadeh proposed the FSs to describe the uncertainty of some specific properties of a given element in the real world [1], and then Zadeh extended FSs to type-2 fuzzy sets and type- $n$ fuzzy sets [2] which gave a trouble-free way to form and minimize the effects of uncertainties in rule-base fuzzy system [3]. Antanassov proposed the intuitionistic fuzzy sets (IFSs) permitting the membership to include some hesitant value [4]. Recently, Torra proposed the hesitant fuzzy sets (HFSs) which are another generalization of FSs. When defining the membership of an element, the membership degree may be a set of possible values rather than interval values or some possibility distribution on the possible values [5]. Xia and $\mathrm{Xu}$ carried out a study on information aggregation for HFSs to make decision, and developed hesitant fuzzy operational rules based on interconnection between HFSs and IFSs [6]. Xu and Xia extended a series of distance and similarity measures for HFSs based on the distinguished Hamming distance, Euclidean distance, Hausdorff metric and their generalizations, and also gave three ways to determine the associated weighting vectors which are used in a class of ordered weighted distance measures for HFSs [7]. Zhu et al. proposed the hesitant fuzzy geometric Bonferroni mean with some special discussed cases and properties, and the hesitant fuzzy Choquet geometric Bonferroni mean combined with the Choquet integral, and furthermore applied a weighted hesitant fuzzy geometric Bonferroni mean to multicriteria decision making [8]. Wei developed some hesitant fuzzy prioritized operators, i.e. prioritized weighted average operator and prioritized weighted geometric operator for HFSs to solve the decision making problem with the hesitant fuzzy multiple attributes which were at different priority levels [9]. Yu et al. extended the generalized Bonferroni mean for HFSs and proposed the generalized hesitant fuzzy Bonferroni mean [10]. Rodriguez et al. introduced the hesitant fuzzy linguistic term sets to make linguistic elicitation more flexible and abundant to support the elicitation of linguistic information supplied by experts, and presented some computational functions and properties for hesitant fuzzy linguistic term sets [11]. Zhu et al. proposed the dual hesitant fuzzy sets (DHFSs), which had two sets (membership degree sets and nonmembership degree sets) to provide more information and more flexible ways for decision maker, and also developed some necessary operations and properties for DHFSs [12].

Fuzzy logic is significant to human decision-making and has been applied to various computer image processing. Tobias proposed an approach to threshold the histogram between gray levels according to fuzzy similarity overcome the local minima [13]. Van presented a new fuzzy filter for the noise reduce of image corrupted with additive noise [14]. Mitra and Pal managed to concluded the functions of fuzzy sets for image processing, pattern recognition, and machine intelligence [15]. Tamalika and Ray proposed a new distance measure named intuitionistic fuzzy divergence to carry out edge detection [16]. Andre and Olivier studied a new fuzzy image filter controlled by interval-valued fuzzy sets for removing noise from images to restrict the number of thresholds or parameters which have to be tuned [17]. Khang and Nor presented a fuzzy approach called histogram thresholding - fuzzy C-means hybrid (HTFCM) to find different applicaton in pattern recognition and especially in color segmentation [18]. Om et al. investigated the fuzzy logic and 
used the fuzzy derivative and bacterial foraging algorithm to propose a new edge detection measure to deal with the noisy image [19]. Dillip and Ashutosh presented an improved membership function was used to fuzzify the image matrix, and then the fuzzy sets logic and mathematical morphological operations to enhance the contrast of images [20]. Unfortunately, there have been few papers of FSs focusing on the comprehensive assessment of image features and recognition. In this paper, we introduce a distance-based similarity measure of DHFSs and propose an comprehensive assessment method of image comparison based on the distancebased similarity measure, where five criteria are represented by DHFSs to assess the similarty to determine the ranking order for six alternative images.

The remaining of this paper is organized as follows. In the next section, we briefly introduce fuzzy sets, hesitant fuzzy sets, dual hesitant fuzzy sets and distance-based similarity measure for hesitant fuzzy sets. Section 3 proposes an improved distance-based similarity measure of dual hesitant fuzzy sets. Section 4 solves dual hesitant fuzzy decisionmaking problems by an example to illustrate the effectiveness of the proposed measure. The paper is concluded and present more future work in Section 5.

\section{PRELIMINARIES}

In this section, we review the fuzzy set, hesitant fuzzy sets, dual hesitant fuzzy sets and distance-based similarity measure, which will be needed in the analysis of the following sections.

\subsection{Fussy Sets}

The definition of a fuzzy set from Zadeh's definition is:

Definition 1. Zadeh (1965) defined a fuzzy set $A$ in the universe of discourse $X$ as follows:

$$
A=\left\{\left\langle x, \mu_{A}(x)\right\rangle \mid x \in X\right\},
$$

which is characterized by membership function $\mu_{A}(x): X \rightarrow$ $[0,1]$, where $\mu_{A}(x)$ indicates the membership degree of the element $x$ to the set $A$.

\subsection{Hesitant Fuzzy Sets}

A hesitant fuzzy set is defined in terms of the union of the union of the memberships of a set of fuzzy sets. This definition allows us to represent the output of the hesitation fuzzy set with fuzzy rules based system.

Definition 2. Let $M=\left\{\mu_{1}, \ldots, \mu_{N}\right\}$ be a set of $N$ membership functions. Then, the hesitant fuzzy set associated with $M$, that is $h_{M}$, is defined as follows:

$h_{M}(x)=\bigcup_{u \in M}\{u(x)\}$

The lower and upper bound of an assumptive hesitant fuzzy set hare:

Lower bound: $h^{-}(x)=\min h(x)$ and
Upper bound: $\mathrm{h}^{+}(x)=\max h(x)$.

Obviously, an intuitionistic fuzzy set can be defined by the pair of functions $h^{-}$and $1-h^{+}$.

The basic operations of hesitant fuzzy sets are described as follows, and first of all there is the complement of hesitant fuzzy sets.

Definition 3. Let $h$ be the membership function of a hesitant fuzzy set, and then its complement is defined as follows:

$h^{c}(x)=\bigcup_{\gamma \in h(x)}\{1-\gamma\}$,

And the complement is involutive, i.e.,

$\left(h^{c}\right)^{c}=h$,

Which means $1-(1-\gamma)=\gamma$ for all $\gamma \in h(x)$.

Before defining the union and intersection of hesitant fuzzy sets, the $\alpha$-upper and $\alpha$-lower bounds.

$\alpha$-upper bound: $h_{\alpha}^{+}(x)=\{h \in h(x) \mid h \geq \alpha\}$ and

$\alpha$-lower bound: $h_{\alpha}^{-}(x)=\{h \in h(x) \mid h \leq \alpha\}$.

Definition 4. Let $h_{1}, h_{2}$ be the membership functions of two hesitant fuzzy sets respectively, and then their union represented by as

$\left(h_{1} \cup h_{2}\right)(x)=\left\{h \in\left(h_{1}(x) \cup h_{2}(x)\right) \mid h \geq \max \left(h_{1}^{-}, h_{2}^{-}\right)\right\}$,

or equivalently,

$\left(h_{1} \cup h_{2}\right)(x)=\left(h_{1}(x) \cup h_{2}(x)\right)_{\alpha}^{+}$for $\left.\alpha=\max \left(h_{1}^{-}, h_{2}^{-}\right)\right)$.

Definition 5. Let $h_{1}, h_{2}$ be the membership functions of two hesitant fuzzy sets respectively, and then their intersection represented by $h_{1} \cap h_{2}$ as

$h_{1} \cap h_{2}(x)=\left\{h \in\left(h_{1}(x) \bigcup h_{2}(x)\right) \mid h \leq \min \left(h_{1}^{+}, h_{2}^{+}\right)\right\}$,

or, equivalently,

$h_{1} \cap h_{2}(x)=\left(h_{1}(x) \bigcap h_{2}(x)\right)_{\alpha}^{-}$for $\alpha=\min \left(h_{1}^{+}, h_{2}^{+}\right)$.

\subsection{Dual Hesitant Fuzzy sets}

Dual hesitant fuzzy sets provide more information including two functions with the set of membership values and the set of nonmembership values to make decision. The definition is as follow.

Definition 6. Let $X$ be an assumptive set, and then a dual hesitant fuzzy set $D$ on $X$ is defined as:

$D=\{\langle x, h(x), g(x)\rangle x \in X\}$

In the above statement, $h(x)$ and $g(x)$ are two sets of some values in $[0,1]$, representing the possible membership degrees and monmembership degrees of the element $x \in X$ to the set $D$. There are some conditions: 
$0 \leq \gamma, \eta \leq 1,0 \leq \gamma^{+}+\eta^{+} \leq 1$

where

$\gamma \in h(x), \eta \in g(x), \gamma^{+} \in h^{+}(x)=\bigcup_{\gamma \in h(x)} \max \{\gamma\}$,

and

$\eta^{+} \in g^{+}(x)=\bigcup_{\eta \in g(x)} \max \{\eta\}$.

With these condition above, a dual hesitant fuzzy element is defined as a pair $d(x)=(h(x), g(x))$ represented by $d=(h$, $g)$. Based on the definition, some special dual hesitant fuzzy elements are given as follows:

(1) Complete uncertainty: $d=\{\{0\},\{1\}\}$;

(2) Complete certainty: $d=\{\{1\},\{0\}\}$;

(3) Complete ill-known (all is possible): $d=[0,1]$;

(4) Nonsensical element: $d=\phi(h=\phi, g=\phi)$.

Some results in special case can be obtained depending on the previous knowledge on fuzzy sets system. If $d \neq 0, \gamma+$ $\eta<1$, and $h$ and $g$ have only one value $\gamma$ and $\eta$, a dual hesitant fuzzy set is degraded to an intuitionistic fuzzy set. If $d \neq$ $0, \gamma+\eta=1$, and $h$ and $g$ have only one value $\gamma$ and $\eta$ or $h$ have one value and $g=\phi$, a dual hesitant fuzzy set is degraded to a fuzzy set. If $h \neq \phi, g=\phi$, a dual hesitant fuzzy set is degraded to a hesitant fuzzy set.

In a typical dual hesitant fuzzy set, $\mathrm{h}$ and $\mathrm{g}$ is represented by two intervals as:

$h=\left[\gamma^{-}, \gamma^{+}\right], g=\left[\eta^{-}, \eta^{+}\right]$.

An intuitionistic fuzzy set can be transformed to a hesitant fuzzy set, so $g(x)$ can be transformed to $h^{2}=\left[1-\eta^{+}, 1-\right.$ $\left.\eta^{-}\right]$denoting the membership degrees. The $d(x)$ can be represented with a "nested interval" as follows:

$d=\left[\left[\gamma^{-}, \gamma^{+}\right],\left[1-\eta^{+}, 1-\eta^{-}\right]\right]$.

In terms of fuzzy numbers or interval numbers, the common definition to represent a dual hesitant fuzzy set $d$ is described as follows:

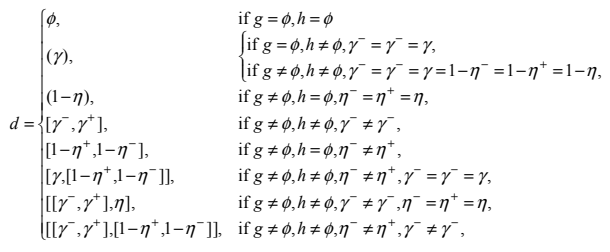

\subsection{Distance and Similarity Measures for Hesitant Fuzzy Sets}

Distance and similarity measures are often used to the similarity for fuzzy sets in the various research fields including pattern recognition, machine learning and other decision making.

Definition 7. Let $A$ and $B$ be two fuzzy sets on $X=\left\{x_{1}\right.$, $\left.x_{2} \ldots x_{n}\right\}$ and $\mu_{A}\left(x_{i}\right)$ and $\mu_{B}\left(x_{i}\right)$ be the membership functions of $A$ and $B$ respectively with the condition $0 \leq \mu_{A}\left(x_{i}\right)$ and $\mu_{B}\left(x_{i}\right) \leq 1$, and $x_{i} \in X$ and $i=1,2, \ldots n$. The commonly used distance measures for $A$ and $B$ are as follows:

$$
\begin{aligned}
& d_{h}(A, B)=\sum_{i=1}^{n}\left|\mu_{A}\left(x_{i}\right)-\mu_{B}\left(x_{i}\right)\right| ; \\
& d_{n h}(A, B)=\frac{1}{n} \sum_{i=1}^{n}\left|\mu_{A}\left(x_{i}\right)-\mu_{B}\left(x_{i}\right)\right| ; \\
& d_{e}(A, B)=\left(\sum_{i=1}^{n}\left|\mu_{A}\left(x_{i}\right)-\mu_{B}\left(x_{i}\right)\right|^{2}\right)^{1 / 2} ; \\
& d_{n e}(A, B)=\frac{1}{n}\left(\sum_{i=1}^{n}\left|\mu_{A}\left(x_{i}\right)-\mu_{B}\left(x_{i}\right)\right|^{2}\right)^{1 / 2} ; \\
& d_{h d}(A, B)=\max \left|\mu_{A}\left(x_{i}\right)-\mu_{B}\left(x_{i}\right)\right| .
\end{aligned}
$$

In the aforementioned definition, Eq. (13) is The Hamming distance; Eq. (14) is the normalized Hamming distance; Eq. (15) is the Euclidean distance; Eq. (16) is the normalized Euclidean distance; and Eq. (17) is the Hausdorff metric.

\section{SIMILARITY MEASURES BASED ON DISTANCE OF DUAL HESITANT FUZZY SETS}

With the definition of hesitant fuzzy sets and the normalized Hamming distance, the distance and similarity measures for hesitant fuzzy sets can be obtained.

Definition 8. Let $M$ and $N$ be two hesitant fuzzy sets on $X=\left\{x_{1}, x_{2} \ldots x_{n}\right\}$, and $E=\left\{\left\langle x, h_{E}(x)\right\rangle \mid x \in X\right\}$ is called the hesitant fuzzy element. The generalized normalized distance for hesitant fuzzy sets is defined as follows:

$d_{\lambda}(M, N)=\left[\frac{1}{n} \sum_{i=1}^{n}\left(\frac{1}{l_{x_{i}}} \sum_{j=1}^{l_{x_{i}}}\left|h_{M}^{\sigma(j)}\left(x_{i}\right)-h_{N}^{\sigma(j)}\left(x_{i}\right)\right|^{\lambda}\right)\right]^{1 / \lambda}$,

In which, $l_{x_{i}}$ is the maximum between $l\left(h_{M}\left(x_{i}\right)\right)$ and $l\left(h_{N}\left(x_{i}\right)\right)$ for each $x_{i}$ in $X$, and and are the $j$ th largest values $h_{M}\left(x_{i}\right)$ and $h_{N}\left(x_{i}\right)$ respectively.

For all $M, N$, the distance measure and similarity measure between $M$ and $N$ is defined as $d(M, N)$ and $s(M, N)$, which satisfies the following properties:

(1) $s(M, N)=1-d(M, N)$;

(2) $0 \leq d(M, N), s(M, N) \leq 1$;

(3) $d(M, N)=0$ or $s(M, N)=1$ if and only if $M=N$;

(4) $d(M, N)=d(N, M), s(M, N)=s(N, M)$;

In terms of dual hesitant fuzzy sets and distance similarity measure, we propose the Similarity measures based on distance of dual hesitant fuzzy sets.

Definition 9. Let $M$ and $N$ be two dual hesitant fuzzy sets on $X=\left\{x_{1}, x_{2} \ldots x_{n}\right\}$, and $E=\left\{\left\langle x,\left(h_{E}(x), g_{E}(x),\right)\right\rangle \mid x \in X\right\}$ is called the dual hesitant fuzzy element. The generalized normalized distance for hesitant fuzzy sets is defined as follows: 


$$
d(M, N)=\left[\frac{1}{2 n}\left(\sum_{i}^{n}\left(\frac{1}{l_{h_{i}}} \sum_{j=1}^{l_{h_{i}}}\left|h_{M}^{\sigma(j)}-h_{N}^{\sigma(j)}\right|^{\lambda}+\frac{1}{l_{g_{i}}} \sum_{k=1}^{l_{g_{i}}}\left|g_{M}^{\sigma(k)}-g_{N}^{\sigma(k)}\right|^{\lambda}\right)\right)\right]^{1 / \lambda},
$$

In which, $l_{h_{i}}$ is the maximum between $l\left(h_{M}\left(x_{i}\right)\right)$ and $l\left(h_{N}\left(x_{i}\right)\right)$ for each $x_{i}$ in $X$, and $l_{g_{i}}$ is the maximum between $l\left(g_{M}\left(x_{i}\right)\right)$ and $l\left(g_{N}\left(x_{i}\right)\right)$ for each $x_{i}$ in $X$, and are the $j$ th largest values $h_{M}\left(x_{i}\right)$ and $h_{N}\left(x_{i}\right)$, and and are the $k$ th largest values $g_{M}\left(x_{i}\right)$ and $g_{N}\left(x_{i}\right)$ respectively.

If $\lambda=1$, the generalized normalized distance for hesitant fuzzy sets is reduced to the dual hesitant normalized Hamming distance:

$$
d(M, N)=\frac{1}{2 n}\left(\sum_{i}^{n}\left(\frac{1}{l_{h_{i}}} \sum_{j=1}^{l_{h_{i}}}\left|h_{M}^{\sigma(j)}-h_{N}^{\sigma(j)}\right|^{\lambda}+\frac{1}{l_{g_{i}}} \sum_{k=1}^{l_{g_{i}}}\left|g_{M}^{\sigma(k)}-g_{N}^{\sigma(k)}\right|^{\lambda}\right)\right) .
$$

If $\lambda=2$, the generalized normalized distance for hesitant fuzzy sets is reduced to the dual hesitant normalized Eulidean distance:

$$
d(M, N)=\left[\frac{1}{2 n}\left(\sum_{i}^{n}\left(\frac{1}{l_{h_{i}}} \sum_{j=1}^{l_{h_{i}}}\left|h_{M}^{\sigma(j)}-h_{N}^{\sigma(j)}\right|^{\lambda}+\frac{1}{l_{g_{i}}} \sum_{k=1}^{l_{g_{i}}}\left|g_{M}^{\sigma(k)}-g_{N}^{\sigma(k)}\right|^{2}\right)\right]^{1 / 2}\right.
$$

For all $M, N$ belonging to dual hesitant fuzzy sets, the distance measure and similarity measure between $M$ and $N$ is defined as $d(M, N)$ and $s(M, N)$, which satisfies the following properties:

(1) $s(M, N)=1-d(M, N)$;

(2) $0 \leq d(M, N), s(M, N) \leq 1$;

(3) $d(M, N)=0$ or $s(M, N)=1$ if and only if $M=N$;

(4) $d(M, N)=d(N, M), s(M, N)=s(N, M)$;

\section{COMPRESSION ASSESSMENT OF IMAGES}

Let us consider the decision-making problem discussed in [21], which is to solve the problem of the compression assessment of images. There are five assessment criteria with respect to alternative images in the evaluations of image features are given by using dual hesitant fuzzy sets. Suppose that there exists a set of alternative image $A=\left\{A_{1}, A_{2}, \ldots\right.$, $\left.A_{m}\right\}$. Each alternative image is assessed on $n$ criteria denoting by $C=\left\{C_{1}, C_{2}, \ldots, C_{n}\right\}$. The gained value of a criterion $C_{j}(j=1,2, \ldots, n)$ on an alternative image $A_{i}(i=1,2, \ldots, m)$ is a dual hesitant fuzzy set $(i=1,2, \ldots, m ; j=1,2, \ldots, n)$ according to image data of the evaluated criteria. A dual hesitant fuzzy set assessment matrix $D=\left(e_{i j}\right)_{m \times n}$ is obtained, and is defined as follows:

$$
\begin{gathered}
C_{1} \\
A_{1} \\
A_{2} \\
\vdots \\
A_{m}
\end{gathered}\left[\begin{array}{cccc}
{\left[h_{11}, g_{11}\right]} & {\left[h_{12}, g_{12}\right]} & \cdots & {\left[\left[h_{1 n}, g_{1 n}\right]\right]} \\
{\left[h_{21}, g_{21}\right]} & {\left[h_{22}, g_{22}\right]} & \cdots & {\left[h_{2 n}, g_{2 n}\right]} \\
\vdots & \vdots & \vdots & \vdots \\
{\left[h_{m 1}, g_{m 1}\right]} & {\left[h_{m 2}, g_{m 2}\right]} & \cdots & {\left[h_{m n}, g_{m n}\right]}
\end{array}\right]
$$

Before comprehensive assessment of images, an ideal point, which provides a significant theoretical support, is needed to help identify the assessment grades in the alternative image, and an ideal dual hesitant fuzzy set is defined for each criterion in the ideal alternative $A^{*}=\left\{\left[h_{1}^{*}, g_{1}^{*}\right],\left[h_{2}^{*}, h_{2}^{*}\right], \cdots,\left[h_{n}^{*}, h_{n}^{*}\right]\right\}$.

Thus the similarity measure between an alternative image $A_{i}$ and the ideal alternative $A^{*}$ represented by dual hesitant fuzzy sets is given as follows:

$$
S\left(A^{*}, A_{i}\right)=1-\left[\frac{1}{2 n}\left(\sum_{i}^{n}\left(\begin{array}{c}
\frac{1}{l_{h_{i}}} \sum_{j=1}^{l_{h_{i}}}\left|h_{M}^{\sigma(j)}-h_{N}^{\sigma(j)}\right|^{\lambda} \\
+\frac{1}{l_{g_{i}}} \sum_{k=1}^{l_{g_{i}}}\left|g_{M}^{\sigma(k)}-g_{N}^{\sigma(k)}\right|^{\lambda}
\end{array}\right)\right]\right]^{1 / \lambda}
$$

The similarity measure of distance provides the global evaluation for each alternative image regarding the ideal alternative on all the criteria. From Eq. (23), the larger the value of the similarity measure of distance is, the more similar the alternative image is.

\section{PRACTICAL EXAMPLE}

In the following practical example, a problem is involved in computer image recognition to give the assessment ranking of image features in some images. The example considers various criteria involving (1) $C_{1}$ : color; (2) $C_{2}$ : texture; (3) $C_{3}$ : shape; (4) $C_{4}$ : space; (5) $C_{5}$ : structure. The example evaluates six alternative images, $A=\left\{A_{1}, A_{2}, A_{3}, A_{4}, A_{5}, A_{6}\right\}$, based on five criteria, $C=\left\{C_{1}, C_{2}, C_{3}, C_{4}, C_{5}\right\}$. The assessment matrix for the alternative images $A_{i} \in A$ with respect to the criterion $C_{j} \in C$ is given by Table 1 .

In terms of some ideal situation, an ideal dual hesitant fuzzy set is defined for criteria in the ideal image as:

$A^{*}=\{\{\{0.97,0.96,0.95\},\{0.03,0.02,0.01\}\},\{\{0.97$, $0.96,0.95\},\{0.03,0.02,0.01\}\},\{\{0.90,0.85,0.80\},\{0.03$, $0.02,0.01\}\},\{\{0.90,0.80\},\{0.09,0.05\}\},\{\{0.90,0.60\}$, $\{0.09,0.02\}\}$.

By applying Eq. (23), the similarities on distance are be obtained between an alternative place $A_{i}$ and the ideal alternative $A^{*}$ in Table $2 .:$

Therefore, according to the assessment results in Table 2, the assessment ranking result for all the alternative places are given in Table $\mathbf{3}$.

\section{CONCLUSION}

In this paper, we have provided the similarity measure based on distance of dual hesitant fuzzy sets, and then a practical example was demonstrated in image feature comparison and recognition. In the demonstration, alternative images have a set of criteria represented by dual hesitant fuzzy sets. As was to be expected, the assessment ranking of 
Table 1. Assessment Matrix $D$.

\begin{tabular}{|c|c|c|c|c|c|}
\hline $\boldsymbol{A}_{\boldsymbol{i}}$ (Alternative Images) & $\boldsymbol{C}_{\mathbf{1}}$ : Color & $\boldsymbol{C}_{2}$ : Texture & $\boldsymbol{C}_{\mathbf{3}}$ : Shape & $\boldsymbol{C}_{\mathbf{4}}$ : Space & $\boldsymbol{C}_{\mathbf{5}}$ : Structure \\
\hline \hline \multirow{2}{*}{$A_{1}$} & $\{\{0.85,0.83,0.80\}$, & $\{\{0.33,0.31,0.30\}$, & $\{\{0.70,0.65,0.60\}$, & $\{\{0.60,0.40\}$, & $\{\{0.60,0.30\}$, \\
& $\{0.14,0.12,0,10\}\}$ & $\{0.64,0.62,0.60\}\}$ & $\{0.30,0.25,0.20\}\}$ & $\{0.35,0.30\}\}$ & $\{0.30,0.20\}\}$ \\
\hline \multirow{2}{*}{$A_{2}$} & $\{\{0.65,0.60,0.55\}$, & $\{\{0.03,0.02,0.01\}$, & $\{\{0.35,0.30,0.25\}$, & $\{\{0.60,0.40\}$, & $\{\{0.30,0.10\}$, \\
& $\{0.34,0.32,0.30\}\}$ & $\{0.96,0.95,0.93\}\}$ & $\{0.64,0.62,0.60\}\}$ & $\{0.36,0.32\}\}$ & $\{0.60,0.55\}\}$ \\
\hline \multirow{2}{*}{$A_{3}$} & $\{\{0.85,0.80,0.75\}$, & $\{\{0.55,0.52,0.50\}$, & $\{\{0.60,0.57,0.55\}$, & $\{\{0.95,0.85\}$, & $\{\{0.30,0.10\}$, \\
& $\{0.15,0.13,0.11\}\}$ & $\{0.44,0.43,0.40\}\}$ & $\{0.38,0.35,0.32\}\}$ & $\{0.04,0.03\}\}$ & $\{0.65,0.60\}\}$ \\
\hline \multirow{2}{*}{$A_{4}$} & $\{\{0.80,0.73,0.56\}$, & $\{\{0.50,0.47,0.45\}$, & $\{\{0.15,0.12,0.10\}$, & $\{\{0.80,0.60\}$, & $\{\{0.30,0.10\}$, \\
& $\{0.17,0.14,0.12\}\}$ & $\{0.45,0.43,0.40\}\}$ & $\{0.82,0.77,0.72\}\}$ & $\{0.19,0.14\}\}$ & $\{0.63,0.52\}\}$ \\
\hline \multirow{2}{*}{$A_{5}$} & $\{\{0.90,0.89,0.88\}$, & $\{\{0.84,0.74,0.66\}$, & $\{\{0.80,0.75,0.70\}$, & $\{\{0.60,0.40\}$, & $\{\{0.30,0.10\}$, \\
& $\{0.08,0.06,0.05\}\}$ & $\{0.13,0.12,0.09\}\}$ & $\{0.18,0.15,0.11\}\}$ & $\{0.38,0.31\}\}$ \\
\multirow{2}{*}{$A_{6}$} & $\{\{0.85,0.82,0.80\}$, & $\{\{0.95,0.78,0.66\}$, & $\{\{0.70,0.69,0.68\}$, & $\{\{0.60,0.40\}$, & $\{\{0.30,0.10\}$, \\
& $\{0.14,0.11,0.07\}\}$ & $\{0.03,0.02,0.01\}\}$ & $\{0.28,0.25,0.24\}\}$ & $\{0.35,0.33\}\}$ \\
\hline
\end{tabular}

Table 2. Assessment Results.

\begin{tabular}{|c|c|c|c|c|c|c|}
\hline$\lambda$ & $\mathbf{S}\left(\mathbf{A}^{*}, \mathbf{A}_{\mathbf{1}}\right)$ & $\mathbf{S}\left(\mathbf{A}^{*}, \mathbf{A}_{\mathbf{2}}\right)$ & $\mathbf{S}\left(\mathbf{A}^{*}, \mathbf{A}_{\mathbf{3}}\right)$ & $\mathbf{S}\left(\mathbf{A}^{*}, \mathbf{A}_{\mathbf{4}}\right)$ & $\mathbf{S}\left(\mathbf{A}^{*}, \mathbf{A}_{\mathbf{5}}\right)$ & $\mathbf{S}\left(\mathbf{A}^{*}, \mathbf{A}_{\mathbf{6}}\right)$ \\
\hline \hline$\lambda=1$ & 0.6990 & 0.4633 & 0.7078 & 0.5928 & 0.7638 & 0.7512 \\
\hline$\lambda=2$ & 0.6509 & 0.4170 & 0.6518 & 0.5312 & 0.7018 & 0.6937 \\
\hline$\lambda=6$ & 0.5191 & 0.2748 & 0.5539 & 0.4145 & 0.5747 & 0.5754 \\
\hline$\lambda=10$ & 0.4656 & 0.2041 & 0.5125 & 0.3632 & 0.5261 & 0.5264 \\
\hline
\end{tabular}

Table 3. Assessment Ranking Results.

\begin{tabular}{|c|c|}
\hline$\lambda$ & Ranking \\
\hline \hline$\lambda=1$ & $\mathrm{~A}_{5}>\mathrm{A}_{6}>\mathrm{A}_{3}>\mathrm{A}_{1}>\mathrm{A}_{4}>\mathrm{A}_{2}$ \\
\hline$\lambda=2$ & $\mathrm{~A}_{5}>\mathrm{A}_{6}>\mathrm{A}_{3}>\mathrm{A}_{1}>\mathrm{A}_{4}>\mathrm{A}_{2}$ \\
\hline$\lambda=6$ & $\mathrm{~A}_{6}>\mathrm{A}_{5}>\mathrm{A}_{3}>\mathrm{A}_{1}>\mathrm{A}_{4}>\mathrm{A}_{2}$ \\
\hline$\lambda=10$ & $\mathrm{~A}_{6}>\mathrm{A}_{5}>\mathrm{A}_{3}>\mathrm{A}_{1}>\mathrm{A}_{4}>\mathrm{A}_{2}$ \\
\hline
\end{tabular}

all the alternative images can be given on the basis of the proposed method and is proved to be effective and practical in the process of the assessment process. In addition, the proposed technology can give a more useful way to provide appropriate assessment reference for decision-makers or experts. The proposed assessment method not only provides a new reasonable improvement to the existing assessment methods, but also simply develops an increasingly wide utilization of dual hesitant fuzzy sets in a new applied field.

In the future, we shall continue to improve the proposed method to solve more complex assessment problems, and extend to other application domains.

\section{CONFLICT OF INTEREST}

The authors confirm that this article content has no conflicts of interest.

\section{ACKNOWLEDGEMENTS}

This paper belongs to the project of the "Special training program for the construction of teachers of Beijing higher education-2014 training project for senior visiting scholars of young and middle-aged excellent teachers" No. 067145301400 . 


\section{REFERENCES}

[1] L. A. Zadeh, "Fuzzy sets," Information and Control, vol. 8, pp. 338-356, 1965.

[2] L. A. Zadeh, "The concept of a linguistic variable and its application to approximate reasoning," I. Inform. Sciences, vol. 8(3), pp. 199-249, 1975.

[3] M. M. Jerry and I. B. J. Robert, "Type-2 fuzzy sets made simple," IEEE T. Fuzzy Syst., vol. 10, pp. 117-127, 2002.

[4] K. Antanassov, "Intuitionistic fuzzy sets," Fuzzy Set Syst., vol. 20, pp. 87-96, 1986.

[5] V. Torra, "Hesitant Fuzzy Sets," Int. J. Intell. Syst., vol. 25, pp. 529-539, 2010.

[6] M. Xia and Z. Xu, "Hesitant fuzzy information aggregation in decision making," Int. J. Approx. Reason., vol. 52, pp. 395-407, 2011.

[7] M. Xia and Z. Xu, "Distance and similarity measures for hesitant fuzzy sets," Inform. Sciences, vol. 181, pp. 2128-2138, 2011.

[8] B. Zhu, Z. Xu and M. Xia, "Hesitant fuzzy geometric Bonferroni means," Inform. Sciences, vol. 205, pp. 72-85, 2012.

[9] G. Wei, "Hesitant fuzzy prioritized operators and their application to multiple attribute decision making," Knowl-Based Syst., vol. 31, pp. 176-182, 2012.

[10] D. Yu, Y. Wu and W. Zhou, "Generalized hesitant fuzzy Bonferroni mean and its application in multi-criteria group decision making," Journal of Information and Com., vol. 9(2), pp. 267-274, 2012.

[11] R. M. Rodriguez, L. Martinez and F. Herrera, "Hesitant Fuzzy Linguistic Term Sets for Decision Making," IEEE T. Fuzzy Syst., vol. 20(1), pp. 109-119, 2012.
[12] B. Zhu, Z. Xu and M. Xia, "Dual hesitant fuzzy sets," Journal of Applied Mathematic, doi:10.1155/2012/879629, 2012.

[13] O. J. Tobias, "Image segmentation by histogram thresholding using fuzzy sets," IEEE Transactions on Image Processing, vol. 11(21), pp. 1457-1465, 2002.

[14] D. V. D. Van, "Noise reduction by fuzzy image filtering," IEEE Transactions on Fuzzy Systems, vol. 11(4), pp. 429-436, 2003.

[15] S. Mitra and S. K. Pal, "Fuzzy sets in pattern recognition and machine intelligence," Fuzzy Sets and Systems, vol. 156(3), pp. 381$386,2005$.

[16] C. Tamalika and A. K. Ray, "A new measure using intuitionistic fuzzy set theory and its application to edge detection," Applied Soft Computing, vol. 8(2), pp. 919-927, 2008.

[17] B. Andre and C. Olivier, "Fuzzy filter based on interval-valued fuzzy sets for image filtering," Fuzzy Sets and Systems, vol. 161(1), pp. 96-117, 2010.

[18] S. T. Khang and A. M. I. Nor, "Color image segmentation using histogram thresholding - Fuzzy C-means hybrid approach," Pattern Recognition, vol. 44(1), pp. 1-15, 2011.

[19] P. K. Om, H. Madasu, K. S. Ashish and S. P. Anil, "A novel fuzzy system for edge detection in noisy image using bacterial foraging," Multidimensional Systems and Signal Processing, vol. 24(1), pp. 181-198, 2013.

[20] R. N. Dillip and B. Ashutosh, "Image enhancement using fuzzy morphology," Journal of Engineering Computers \& Applied Sciences, vol. 3(3), pp. 22-26, 2014.

(C) Chen and Bai; Licensee Bentham Open.

This is an open access article licensed under the terms of the Creative Commons Attribution Non-Commercial License (http://creativecommons.org/licenses/by-nc/3.0/) which permits unrestricted, non-commercial use, distribution and reproduction in any medium, provided the work is properly cited. 